\title{
19. University
}

Kemi, O. J., Fowler, E., Mcglynn, K., Primrose, D., Smirthwaite, R. and Wilson, J. (2019) Intensity-dependence of exercise and active recovery in high-intensity interval training. Journal of Sports Medicine and Physical Fitness, 59(1), pp. 1937-1943. (doi: 10.23736/S0022-4707.19.09521-5)

The material cannot be used for any other purpose without further permission of the publisher and is for private use only.

There may be differences between this version and the published version. You are advised to consult the publisher's version if you wish to cite from it.

\section{$\underline{\text { http://eprints.gla.ac.uk/183667/ }}$}

Deposited on 08 April 2019

Enlighten - Research publications by members of the University of

Glasgow

http://eprints.gla.ac.uk 
Intensity-dependence of exercise and active recovery in high-intensity interval training

Ole J. Kemi ${ }^{1 *}$, Ewan Fowler ${ }^{1}$, Karen McGlynn ${ }^{1}$, Deborah Primrose ${ }^{1}$, Rachel Smirthwaite ${ }^{1}$, John Wilson ${ }^{1}$

${ }^{1}$ School of Life Sciences and Institute of Cardiovascular and Medical Sciences; College of Medical, Veterinary and Life Sciences; University of Glasgow, Glasgow, UK.

*Corresponding author: Dr Ole J Kemi, Sir James Black Building, University of Glasgow, Glasgow, G12 8QQ, UK. E-mail: ole.kemi@glasgow.ac.uk; Phone: +44 (0)141 3305962 


\begin{abstract}
BACKGROUND: High-intensity interval training (HIIT) with interspersing active recovery is an effective mode of exercise training in cohorts ranging from athletes to patients. Here, we assessed the intensity-dependence of the intervals and active recovery bouts for permitting a sustainable HIIT protocol.
\end{abstract}

METHODS: 14 males completed 4x4-minute HIIT protocols where intensities of intervals ranged $80-100 \%$ of maximal oxygen uptake $\left(\mathrm{VO}_{2 \max }\right)$ and active recovery ranged $60-100 \%$ of lactate $\left(\mathrm{La}^{-}\right)$ threshold (LT). Blood La' measurements indicated fatigue, while tolerable duration of intervals indicated sustainability.

RESULTS: HIIT at $100 \%$ of $\mathrm{VO}_{2 \max }$ allowed $44 \pm 10 \%$ [30-70\%] completion, i.e. fatigue occurred after 7minutes:6seconds of the intended 16 minutes of high intensity, whereas HIIT at $95-80 \%$ of $\mathrm{VO}_{2 \max }$ was $100 \%$ sustainable $(p<0.01)$. Measured intensity did not differ from intended intensity across the protocols $(p>0.05)$. Blood $\mathrm{La}^{-}$concentration [ $\left.\mathrm{La}^{-}\right]$increased to $9.3 \pm 1.4 \mathrm{mM}$ during HIIT at $100 \%$ of $\mathrm{VO}_{2 \max }$, whereas at $80-95 \%$ of $\mathrm{VO}_{2 \max }$ stabilised at $2-6 \mathrm{mM}$ in an intensity-dependent manner ( $p<0.01$ vs $100 \%$ of $\mathrm{VO}_{2 \max }$ and $p<0.05$ vs baseline). Active recovery at $60-70 \%$ of LT during HIIT associated with steady-state blood $\left[\mathrm{La}^{-}\right]$peaking at $6-7 \mathrm{mM}$, whereas at $80-100 \%$ of LT, blood $\left[\mathrm{La}^{-}\right]$accumulated to $10-13 \mathrm{mM}(p<0.05)$. After HIIT, active recovery at $80-90 \%$ of LT cleared blood $\left[\mathrm{La}^{-}\right] 90 \%$ faster than at $60-70 \%$ of LT $(p<0.05)$.

CONCLUSIONS: To permit highest exercise stress during 4x4-minute HIIT, exercise intensity should be set to $95 \%$ of $\mathrm{VO}_{2 \max }$, whereas active recovery should be set to $60-70 \%$ of LT during HIIT and $80-90 \%$ of LT after HIIT to most efficiently prevent excess $\mathrm{La}^{-}$and aid recovery.

Key words: High-intensity interval training, Endurance, Active recovery, Fatigue, Lactate. 


\section{TEXT}

\section{Introduction}

High-intensity interval training (HIIT) in the form of $4 \times 4$-minute intervals with interspersed recovery bouts, is a popular and effective form of exercise training that has been adopted widely by many different cohorts ranging from athletes to patients ${ }^{1-3}$ and that in many instances accrues greater benefits than moderate-intensity exercise. 4x4-minute HIIT performed at $90-95 \%$ of maximum heart rate $(\mathrm{HR})$ is significantly more effective for increasing maximal oxygen uptake $\left(\mathrm{VO}_{2 \max }\right)$ and cardiac contractile parameters, ${ }^{3}$ as well as performance ${ }^{2}$ than exercise at $70 \%$ of maximum HR, even after matching for energy consumption. For peripheral and skeletal muscle adaptations, similar intensity-dependence of effects has generally been observed, albeit to a lesser extent or with less confidence. ${ }^{4}$ However, although the intensity-dependence of effect has been accepted as a principle, the highest intensity of HIIT that may be achieved and completed has not yet been reported.

Moreover, because the intensity of HIIT is above lactate ( $\left.\mathrm{La}^{-}\right)$threshold (LT) $)^{5,6}$ or critical velocity, ${ }^{7}$ it is limited by early onset of fatigue that coincides with metabolite accumulation, including $\mathrm{La}^{-}$. This is repelled by active recovery bouts interspersing the high-intensity intervals, which allow for accumulation of high intensity beyond that achieved by each individual 4-minute bout, but the optimal active recovery intensity during HIIT remains unknown.

Thus, the benefit of HIIT is firstly restricted by the balance between exercising at the highest sustainable intensity for the duration of the work period; too high intensity limits duration, and too low intensity, though sustainable, carries less adaptation. Secondly, the degree of recovery achieved during the active recovery bouts also affects the outcome. As such, finding this balance becomes important to optimize the outcome of HIIT.

Here, we determined the highest sustainable intensity that allows completion of a full $4 \times 4$-minute HIIT protocol, and subsequently assessed intensity-dependence of active recovery for removing $\mathrm{La}^{-}\left(\mathrm{La}^{-}\right.$in this context being a marker of fatigue $\left.{ }^{5,6}\right)$ during HIIT to thereby identify if an optimal intensity of active recovery during 4x4-minute HIIT exists. 


\section{Materials and Methods}

\section{Subjects}

14 moderately active males volunteered for participation, with characteristics as follows (mean $\pm \mathrm{SD}$ ): $23.8 \pm 3.0$ years, $180.2 \pm 5.7 \mathrm{~cm}$ and $70.5 \pm 7.0 \mathrm{~kg}$; all were health screened and signed informed consent forms prior to commencement. Exclusion criteria were regular smoking, medication, drugs, and pre-existing medical conditions contraindicative to exercise testing, as well as avoidance of exhaustive exercise and alcohol within 48 hours and food and fluids except water within 2 hours of each test. The Institutional Review Board (University of Glasgow College of Medical Veterinary and Life Sciences Ethics Committee, Chair Professor W Martin, \#1006, 13/09/2013) approved the study and it conformed to the Declaration of Helsinki.

\section{Procedures}

We designed 2 repetitive HIIT protocols to identify optimal intensity and recovery. To minimize training effects during participation of the study, each subject completed only 1 protocol following randomization. As such, subjects were randomized to either of the exercise intensity of HIIT ( $n=7$ ) or the active recovery intensity of HIIT $(n=7)$ protocols. Within each protocol, subjects completed 2 incremental exercise tests and 5 HIIT tests (see below for details), with at least 48 hours separating each test.

\section{Incremental Exercise Tests}

Subjects warmed-up by a 10-minute, $8 \mathrm{~km} / \mathrm{h}$ flat treadmill run (PPS55 Med, Woodway, Birmingham, UK). A ramped incremental exercise test measured $\mathrm{VO}_{2 \max }$, with speed increasing 1 $\mathrm{km} / \mathrm{h} /$ minute until volitional exhaustion, with $\mathrm{VO}_{2 \max }$ considered achieved with at least 3 of 4 criteria being met: oxygen uptake $\left(\mathrm{VO}_{2}\right)$ plateau, respiratory exchange ratio $>1.15$, post-exercise $\mathrm{La}^{-}$concentration $\left(\left[\mathrm{La}^{-}\right]\right)>8 \mathrm{mM}$, and HR within $10 \mathrm{bpm}$ of age-predicted maximum. ${ }^{5,6}$

After a similar warm-up, LT was measured with speed increasing $0.5 \mathrm{~km} / \mathrm{h}$ every 4 minutes, until blood [ $\left.\mathrm{La}^{-}\right]$, measured at end of each 4-minute step, increased exponentially. ${ }^{5-7} \mathrm{LT}$ was determined by identifying the deflection point of blood $\mathrm{La}^{-}$increase. ${ }^{8}$

$\mathrm{La}^{-}$was sampled from finger prick capillary blood (Analox GM7 Lactate Analyzer, Analox, Hammersmith, UK), $\mathrm{VO}_{2}$ measured by continuous 1-minute Douglas bag-collected expired gas samples (Servomex 4100 Gas Analyser, Servomex, Sussex, UK), and HR by a chest monitor (Polar Heart Rate FS1, Polar, Kempele, Finland). 
To identify highest sustainable exercise intensity of HIIT, 7 subjects completed a series of 5 randomized HIIT protocols, whereby the 4-minute high-intensity intervals were set to correspond to $80 \%, 85 \%, 90 \%, 95 \%$, or $100 \%$ of $\mathrm{VO}_{2 \max }(\mathrm{ON}$-transients; Figure $1 \mathrm{~A}$ ) for the entire session, with the subjects actively encouraged to complete each protocol as much as possible before volitional exhaustion. $\mathrm{VO}_{2}$ and $\mathrm{HR}$ measurements in the last minute of each interval confirmed the intensity of each ON-transient. 4-minute active recovery bouts (OFF-transients), set to 60\% of LT, interspersed the ON-transients (Figure 1A). Time sustained in the ON-transient was recorded.

\section{Active Recovery Intensity of HIIT}

To identify intensity-dependence of active recovery (OFF-transients) of a HIIT protocol, 7 subjects completed a series of 5 HIIT protocols, whereby the 4-minute high-intensity intervals were set to $95 \%$ of $\mathrm{VO}_{2 \max }$ (as it was identified as the optimal exercise intensity of the ONtransients in the above-mentioned experiments), and the 4-minute active recovery bouts were randomized to correspond to $60 \%, 70 \%, 80 \%, 90 \%$, or $100 \%$ of LT (OFF-transients; Figure 1A) for the entire session, with the last OFF4-transient prolonged to allow full recovery. Blood $\mathrm{La}^{-}$was sampled at the end of each ON-transient and every minute of OFF-transients, whereas ONtransient $\mathrm{VO}_{2}$ was recorded to confirm intensity.

\section{Statistics}

Data are expressed as means \pm standard deviation (SD). One-way analysis of variance (ANOVA) and repeated measures general linear model (GLM) assessed differences between intensities; Scheffe post-hoc tests identified effects. Statistical significance was set to $p<0.05$. For analysis of $\mathrm{La}^{-}$clearance during prolonged active recovery after the HIIT protocol, we normalized $\mathrm{La}^{-}$ removal curves and fitted exponential decay curves to derive time constants (2/3) and computed $1^{\text {st }}$ derivatives for maximal rate of $\mathrm{La}^{-}$clearance, whereby fitted curves were also compared to raw curves by regression. 


\section{Results}

Incremental Exercise Tests

Subjects presented with $\mathrm{VO}_{2 \max } 60.5 \pm 6.0 \mathrm{~mL} / \mathrm{kg} / \mathrm{min}$, maximal HR $198 \pm 4 \mathrm{bpm}$, and LT at running velocity $11.9 \pm 2.0 \mathrm{~km} / \mathrm{h}, \mathrm{VO}_{2} 40.2 \pm 4.2 \mathrm{~mL} / \mathrm{kg} / \mathrm{min}, \% \mathrm{VO}_{2 \max } 66.4 \pm 4.5 \%$, HR $160 \pm 7 \mathrm{bpm}$, and $\% \mathrm{HR}$ maximum $79.3 \pm 6.0 \%$. This corresponds with a moderate to good level of fitness and indicates subjects were regularly physically active.

\section{Exercise Intensity of HIIT}

First, we identified the optimal exercise intensity of the current 4x4-minute HIIT protocol, defining this to be the highest sustainable intensity that allowed completion of the $4 \mathrm{x} 4$-minute ON-transients of a given intensity protocol. We found that when the intensity of the ON-transients was set to $100 \%$ of $\mathrm{VO}_{2 \max }$, only $44 \pm 10 \%$ of the protocol could be completed [range $30-70 \%$ ] with intended intensity, before it dropped and irretrievable (for the session) onset of fatigue occurred (Figure 1B). Thus, fatigue occurred on average after 7 minutes 6 seconds, i.e. 3 minutes 6 seconds into the $2^{\text {nd }}$ ON-transient [range 4 minutes 57 seconds- 10 minutes 51 seconds], of a maximum ON-transient exercise time of 16 minutes. Effectively, this means only 2-3 of 4 ON-transients could be sustained and completed at this intensity. In contrast, HIIT with ON-transients ranging $95-80 \%$ of $\mathrm{VO}_{2 \max }$ were all sustainable for the full duration of the HIIT protocol (Figure 1B).

Exercise intensity during HIIT protocols was measured in order to control that intended intensity was successfully achieved. By measuring $\mathrm{VO}_{2}$ during the $\mathrm{ON}$-transients, we found that the true intensities differed $0.24 \pm 1.1 \%$ from the intended intensities across the various HIIT protocols (80$100 \%$ of $\mathrm{VO}_{2 \max }$ ), with no statistical differences between intended and measured intensities (oneway ANOVA $p>0.05$ ). Furthermore, although intensity was not set by HR, we found that HR during HIIT at $100 \%$ of $\mathrm{VO}_{2 \mathrm{max}}$ reached maximal HR (197 $\pm 3 \mathrm{bpm}$, range 195-202bpm), and in the lower intensities predictably reached values commensurate with the lower HIIT intensities of 80$95 \%$ of $\mathrm{VO}_{2 \max }$ (one-way ANOVA $p<0.05$ between intensities).

Finally, we measured blood [ $\left.\mathrm{La}^{-}\right]$at the end of each ON- and OFF-transient (Figure 1C). This showed that blood [ $\left.\mathrm{La}^{-}\right]$at HIIT intensity of $100 \%$ of $\mathrm{VO}_{2 \max }$ increased to $9.3 \pm 1.4 \mathrm{mM}$ (range 8.2$11.3 \mathrm{mM}$ ) and did not decrease during OFF-transients. In contrast, $\left[\mathrm{La}^{-}\right]$during HIIT intensities 80$95 \%$ of $\mathrm{VO}_{2 \max }$ increased to $2-6 \mathrm{mM}$ in an intensity-dependent manner, but significantly less compared to $100 \% \mathrm{VO}_{2 \max }$-intensity (Figure 1C). On further analysis, blood [ $\left.\mathrm{La}^{-}\right]$decreased during active recovery OFF-transients at HIIT protocols $80-95 \%$ of $\mathrm{VO}_{2 \max }$, whereas during HIIT at $100 \%$ of $\mathrm{VO}_{2 \max }$, it continually increased until further exercise was unsustainable, including during the OFF1-transient. Thus, blood $\left[\mathrm{La}^{-}\right]$accumulation occurred at a rate at least $140 \%$ higher 
during HIIT at $100 \%$ of $\mathrm{VO}_{2 \max }$ compared to the lower intensities of $80-95 \%$ of $\mathrm{VO}_{2 \max }$ $\left(1.05 \pm 0.55 \mathrm{mM} / \mathrm{min}\right.$ vs. $0.23 \pm 0.10 \mathrm{mM} / \mathrm{min}$ at $80 \%$ of $\mathrm{VO}_{2 \max }$ to $0.43 \pm 0.11 \mathrm{mM} / \mathrm{min}$ at $95 \%$ of $\mathrm{VO}_{2 \max }$; Figure $2 \mathrm{~A}$ ). In the $80-95 \%$ of $\mathrm{VO}_{2 \max }$ exercise intensity range, ON-transient blood [ $\left.\mathrm{La}^{-}\right]$ accumulation was matched by active recovery OFF-transient blood $\left[\mathrm{La}^{-}\right]$clearance, whereas at $100 \%$ of $\mathrm{VO}_{2 \max }$, no blood [ $\left.\mathrm{La}^{-}\right]$clearance occurred; in fact, blood [ $\left.\mathrm{La}^{-}\right]$continued to rise during the OFF-transient at this exercise intensity (Figure 2B), to the extent that the total rate of blood $\left[\mathrm{La}^{-}\right]$accumulation (increase during ON-transients - clearance during OFF-transients) increased $\sim 6$ times when increasing HIIT exercise intensity from $95 \%$ to $100 \%$ of $\mathrm{VO}_{2 \max }$; i.e. $0.24 \pm 0.11 \mathrm{mM} / \mathrm{min}$ at $95 \%$ of $\mathrm{VO}_{2 \max }$ to $1.45 \pm 0.44 \mathrm{mM} / \mathrm{min}$ at $100 \%$ of $\mathrm{VO}_{2 \max }$ (Figure $2 \mathrm{C}$ ).

\section{Active Recovery Intensity of HIIT}

Next, we aimed to assess intensity-dependence of active recovery and possibly identify the optimal active recovery intensity during 4-minute OFF-transients of a HIIT protocol; i.e. that which removed blood [ $\left.\mathrm{La}^{-}\right]$fastest. For this, ON-transient exercise intensity was set to $95 \%$ of $\mathrm{VO}_{2 \max }$, as this was the highest sustainable exercise intensity that allowed completion of the HIIT protocol (see above); measured ON-transient intensity corresponded to $94.5 \pm 1.5 \%$ of $\mathrm{VO}_{2 \max }$, $p>0.05$ vs intended intensity. ON-transient HR also corresponded with HR observed at this intensity in the previous set of experiments, whereas blood [ $\left.\mathrm{La}^{-}\right]$increased from $0.97 \pm 0.24 \mathrm{mM}$ after warm-up to $5.68 \pm 0.81 \mathrm{mM}$ during ON1-transient (Figure 3), in line with the previous experiments.

On the basis of successful ON-transients that achieved the intended exercise intensity and the expected rise in blood [ $\left.\mathrm{La}^{-}\right]$, active recovery OFF-transients then varied $60-100 \%$ of LT, with $\mathrm{VO}_{2}$ and HR measurements confirming that intended intensity of active recovery was achieved; measured $\mathrm{VO}_{2}$ and $\mathrm{HR}$ during the OFF-transients differed $0.43 \pm 0.08 \%$ and $0.58 \pm 0.21 \%$, respectively, from the intended values (one-way ANOVA $p>0.05$ ). These trials showed that blood $\left[\mathrm{La}^{-}\right]$tended to increase during each HIIT protocol (in fact also during the first 1-2 minutes of active recovery); however, blood $\left[\mathrm{La}^{-}\right]$continued to increase only significantly at HIIT with active recovery set to $80-100 \%$ of LT, but not $60-70 \%$ of LT (Figure 3 ). In particular, after the initial increase to 5-6mM during $\mathrm{ON} 1$, blood $\left[\mathrm{La}^{-}\right]$continued to increase to reach a peak of $13 \mathrm{mM}$, i.e. an increase of $\sim 130 \%$, after ON4 when active recovery was set to $80 \%$ of LT, whereas during active recovery at $60-70 \%$ of $\mathrm{LT}$, blood $\left[\mathrm{La}^{-}\right]$only increased insignificantly to $6-7 \mathrm{mM}$. In other words, active recovery during HIIT is unable to prevent a continued increase of blood [ $\left.\mathrm{La}^{-}\right]$, but may mitigate if performed at $60-70 \%$ of LT. However, the situation was different during the prolonged OFF4-transient, where active recovery at $80-100 \%$ of LT tended to clear accumulated blood [ $\left.\mathrm{La}^{-}\right]$ 
faster than at $60-70 \%$ of LT, though regardless, it took $>20$ minutes for blood [ $\left.\mathrm{La}^{-}\right]$to return to baseline levels even under the best of circumstances (Figure 3).

To substantiate the latter observation, we investigated blood $\mathrm{La}^{-}$clearance during the prolonged post-interval OFF4-transient closer. First, from the observed $\mathrm{La}^{-}$clearance curves, we fitted exponential decay curves to assess time constants for $2 / 3(67 \%)$ clearance. The fitted curves compared well with the observed curves, with $\mathrm{R}^{2}$-values ranging 0.87-0.99 $(p<0.01)$ and a coefficient of variation of $4 \%$. From peak $\left[\mathrm{La}^{-}\right]$, the time constant for $2 / 3 \mathrm{La}^{-}$clearance was $\sim 90 \%$ longer during active recovery at $60-70 \%$ of LT compared to higher intensities (Figure 4A). We then calculated the $1^{\text {st }}$ derivative of the observed curves in order to assess the maximal rates of $\mathrm{La}^{-}$ clearance. In line with the above, this showed that the maximal rate was $\sim 90 \%$ higher during active recovery at $80-100 \%$ of LT compared to lower intensities (Figure 4B). 


\section{Discussion}

In this study, we aimed to first determine the highest sustainable exercise intensity relative to $\mathrm{VO}_{2 \max }$ that allows completion of a full $4 \mathrm{x} 4$-minute HIIT protocol, and secondly to assess the intensity-dependence of active recovery relative to LT for removing $\mathrm{La}^{-}$during HIIT in order to determine if an optimal intensity of active recovery during HIIT exists, and if so, identify this intensity. Finding the optimal intensities of high-intensity intervals and active recovery bouts becomes important to optimize the outcome of HIIT. As such, the overarching aim was to inform strategies for designing optimal and practically sustainable protocols for high-intensity training by the use of HIIT.

From these experiments, we found:

- HIIT performed with interval intensities (ON-transients) corresponding to $95 \%$ of $\mathrm{VO}_{2 \max }$ permits completion of the full 4x4-minute protocol at the highest intensity, whereas in comparison, HIIT at $100 \%$ of $\mathrm{VO}_{2 \max }$ is not sustainable - only $44 \%$ of protocol was sustained on average and no subject sustained $>70 \%$ of protocol at this intensity - and while exercise intensities $<95 \%$ of $\mathrm{VO}_{2 \max }$ are therefore obviously sustainable, they associate with less exercise stimulus and stress. ${ }^{4}$ Of note, active recovery during these trials was set to $60 \%$ of LT, as it allowed for steady-state active recovery conditions.

- Increasing HIIT ON-transient exercise intensity from $95 \%$ to $100 \%$ of $\mathrm{VO}_{2 \max }$ is associated with a substantial change in blood [ $\left.\mathrm{La}^{-}\right]$dynamics; at the lower intensity it remains relatively steady-state during HIIT after an initial increase, whereas at the higher intensity, it accumulates $>6$ times faster and without any steady-state. This may at least partly explain why an exercise intensity of $100 \%$ of $\mathrm{VO}_{2 \max }$ cannot be sustained in the context of 4x4-minute HIIT protocols.

- Active recovery at $60-70 \%$ of LT in the OFF-transients during 4x4-minute HIIT (ONtransient intensity of $95 \%$ of $\mathrm{VO}_{2 \max }$ ) allows for steady-state blood $\left[\mathrm{La}^{-}\right]$, whereas active recovery at higher intensities is associated with accumulation of blood [ $\left.\mathrm{La}^{-}\right]$; however, this accumulation did still allow for continuation and successful completion of the HIIT protocol.

- For the prolonged OFF-transient after the 4x4-minute HIIT protocol, active recovery at 80$90 \%$ of LT, and to a lesser extent also $100 \%$ of LT, removed $\mathrm{La}^{-}$faster than $60-70 \%$ of $\mathrm{LT}$ active recovery intensities; however, in all cases, at least 20 minutes of active recovery was required to fully remove excess blood $\mathrm{La}^{-}$. 
It should be pointed out that intensity of intervals (ON-transients) and active recovery bouts (OFFtransients) was always confirmed by continuous $\mathrm{VO}_{2}$ and $\mathrm{HR}$ measurements; i.e. the intended intensity was truly achieved in the experiments. Moreover, when investigating the highest sustainable ON-transient exercise intensity of HIIT, active recovery OFF-transient intensity was set to $60 \%$ of LT as it ensured steady-state conditions during active recovery, while reciprocally, when investigating optimal active recovery OFF-transient intensity, ON-transient exercise intensity of HIIT was then set to $95 \%$ of $\mathrm{VO}_{2 \max }$, as this had been identified as the highest sustainable exercise intensity of HIIT. Thus, we applied and confirmed optimum conditions where and when applicable throughout the study.

Thus, assuming i) the well-established concept of intensity-dependence of effects in both health and disease, ${ }^{1-4,9,10}$ dictates that exercise intensity should be as high as achievable, and ii) the mode of exercise training is the also well-established $4 \mathrm{x} 4$-minute HIIT protocol, ${ }^{1-3,9,10}$ these results are the first to suggest that the protocol should be carried out with an ON-transient exercise intensity of $95 \%$ of $\mathrm{VO}_{2 \max }$ and that interspersing active recovery bouts should be carried out with an OFFtransient intensity of $60-70 \%$ of LT during HIIT and $80-90 \%$ of LT after HIIT in order to most efficiently stimulate recovery and prevent and remove excess $\mathrm{La}^{-}$that otherwise is generated by the HIIT protocol.

In the present study, we quantified exercise and active recovery intensities relative to the individual subjects' capacity ( $\mathrm{VO}_{2 \max }$ and LT, respectively), as this or the corresponding energy expenditure converted from gas exchange $\mathrm{VO}_{2}$ measurements (metabolic equivalents (MET)), is the preferred norm in the majority of studies and exercise programs, position stands and guidelines offered by professional and authoritative bodies such as the American College of Sports Medicine (ACSM), and in general the exercise and sports science community. ${ }^{11}$ In this context, relative (\%) rather than absolute quantification allows the intensity to be identified and individualized quickly and effectively, though we acknowledge that another appropriate strategy for setting the intensity especially during ON-transients would be by the velocity-tolerable duration relationship from the critical power or velocity concept ${ }^{7}$ however, since this relationship is hyperbolic, it requires a drastically greater laboratory testing effort, especially for running, which may not always be feasible. ${ }^{12}$

We acknowledge that $\mathrm{La}^{-}$in itself may not fully cause or explain muscle fatigue during intense exercise, though it has been implicated in this process. ${ }^{13-15}$ However, the rationale for the use of $\mathrm{La}^{-}$as a biological marker of fatigue in the current study is that it correlates well with onset and generation of fatigue and therefore serves as an accessible, reproducible, and valid proxy measure of fatigue that may be obtained serially during and after exercise training. ${ }^{5,6} \mathrm{La}^{-}$accumulates 
during intense exercise above LT with changes to the redox state of the exercising muscle that converts pyruvate to $\mathrm{La}^{-}$that subsequently diffuses to the blood stream where it is measured. ${ }^{13-16}$ We previously demonstrated this approach to be useful for assessing fatigue and recovery after single bouts of exercise and showed that blood $\mathrm{La}^{-}$measurements could identify optimal intensity of active recovery during circumstances of both limited and excessive accumulations of $\mathrm{La}^{-}{ }^{5,6} \mathrm{We}$ now demonstrate the same is true for assessing the same parameters during HIIT, but in this case the repetitive accumulation and incomplete clearance due to the cyclical nature of the HIIT protocol adds complexity. As part of this, we observed a discrepancy of $\mathrm{La}^{-}$clearance in this study compared to the previous studies, as after single bouts of exercise, active recovery at $80-100 \%$ of LT consistently cleared La- faster than lower intensities, ${ }^{5,6}$ whereas in the current study, faster La clearance by active recovery was achieved at $60-70 \%$ of LT during HIIT and at $80-90 \%$ of LT after HIIT; the latter during prolonged active recovery and in line with the previous results, which as such confirms that during continued active recovery that exceeds the 4-minute bouts inside a HIIT protocol, $\mathrm{La}^{-}$clearance occurs fastest when active recovery is set close to LT, where continued exercise and muscle contractile work metabolises $\mathrm{La}^{-}$at the fastest rate, mainly by oxidation. ${ }^{16}$ Thus, the observed intensity-dependence of $\mathrm{La}^{-}$clearance during active recovery after HIIT is similar to previous observations, ${ }^{5,6}$ whereas active recovery applied to OFF-transients during HIIT has not yet received scrutiny with respect to intensity-dependence of effect, though active recovery has been reported more beneficial than passive recovery. ${ }^{17}$

\section{Conclusions}

First, we aimed to determine the highest sustainable intensity relative to $\mathrm{VO}_{2 \max }$ that allows completion of a full $4 \times 4$-minute HIIT protocol. We found that a protocol with intervals at an exercise intensity of $95 \%$ of $\mathrm{VO}_{2 \max }$ is the highest sustainable intensity that allows completion of the protocol, whereas an exercise intensity above this level is not sustainable. Completing a HIIT protocol at an exercise intensity of $95 \%$ of $\mathrm{VO}_{2 \max }$ associates with elevated, but steady-state blood $\left[\mathrm{La}^{-}\right]$, whereas an intensity above this associates with continued accumulation of [ $\left.\mathrm{La}^{-}\right]$until onset of fatigue. Secondly, we assessed intensity-dependence of interspersing active recovery bouts for removing $\mathrm{La}^{-}$during and after a HIIT protocol. We found that active recovery intensities of 60$70 \%$ of LT during HIIT and $80-90 \%$ of LT after HIIT serve best to prevent and remove excess La. These findings therefore inform when designing efficient training programs that include $4 \times 4$ minute HIIT protocols. 


\section{RERERENCES}

1. Helgerud J, Hoydal K, Wang E, Karlsen T, Berg P, Bjerkaas M, et al. Aerobic high-intensity intervals improve $\mathrm{VO}_{2 \max }$ more than moderate training. Med Sci Sports Exerc 2007;39:66571.

2. Storen $\mathrm{O}$, Bratland-Sanda $\mathrm{S}$, Haave $\mathrm{M}$, Helgerud J. Improved $\mathrm{VO}_{2 \max }$ and time trial performance with more high aerobic intensity interval training and reduced training volume: a case study on an elite national cyclist. J Strength Cond Res 2012;26:2705-11.

3. Wisloff U, Stoylen A, Loennechen JP, Bruvold M, Rognmo O, Haram PM, et al. Superior cardiovascular effect of aerobic interval training versus moderate continuous training in heart failure patients: a randomized study. Circulation 2007;19:3086-94.

4. MacInnis MJ, Gibala MJ. Physiological adaptations to interval training and the role of exercise intensity. J Physiol 2017;595:2915-30.

5. Devlin J, Paton B, Poole L, Sun W, Ferguson C, Wilson J, et al. Blood lactate clearance after maximal exercise depends on active recovery intensity. J Sports Med Phys Fitness 2014;54:271-8.

6. Menzies P, Menzies C, McIntyre L, Paterson P, Wilson J, Kemi OJ. Blood lactate clearance during active recovery after an intense running bout depends on the intensity of the active recovery. J Sports Sci 2010;28:975-82.

7. Ferguson C, Wilson J, Birch KM, Kemi OJ. Application of the speed-duration relationship to normalize the intensity of high-intensity interval training. PLoS One 2013;8:e76420.

8. Newell J, Higgins D, Madden N, Cruickshank J, Einbeck J, McDonald R. Software for calculating blood lactate endurance markers. J Sports Sci 2007;25:1403-9.

9. Karlsen T, Aamot IL, Haykowsky M, Rognmo O. High intensity interval training for maximizing health outcomes. Prog Cardiovasc Dis 2017;60:67-77.

10. Kemi OJ, Wisloff U. High-intensity aerobic exercise training improves the heart in health and disease. J Cardiopulm Rehabil Prev 2010;30:2-11.

11. Garber CE, Blissmer B, Deschenes MR, Franklin BA, Lamonte MJ, Lee IM, et al. American College of Sports Medicine position stand. Quantity and quality of exercise for developing and maintaining cardiorespiratory, musculoskeletal, and neuromotor fitness in apparently healthy adults: guidance for prescribing exercise. Med Sci Sports Exerc 2011;43:1334-59.

12. Vanhatalo A, Jones AM, Burnley M. Application of critical power in sport. Int J Sports Physiol Perform 2011;6:128-36.

13. Ferguson BS, Rogatzki MJ, Goodwin ML, Kane DA, Rightmire Z, Gladden LB. Lactate metabolism: historical context, prior misinterpretations, and current understanding. Eur J Appl Physiol 2018;118:691-728. 
14. Finsterer J. Biomarkers of peripheral muscle fatigue during exercise. BMC Musculoskelet Disord 2012;13:218.

15. Hogan MC, Gladden LB, Kurdak SS, Poole DC. Increased [lactate] in working dog muscle reduces tension development independent of pH. Med Sci Sports Exerc 1995;27:371-7.

16. Gladden LB. Lactate metabolism: a new paradigm for the third millennium. J Physiol 2004;558:5-30.

17. Abderrahman AB, Rhibi F, Ouerghi N, Hackney AC, Saeidi A, Zouhal H. Effects of recovery mode during high intensity interval training on glucoregulatory hormones and glucose metabolism in response to maximal exercise. J Athl Enhanc 2018;7:10.4172/23249080.1000292.

\section{NOTES}

Conflicts of interest.-The authors certify that there is no conflict of interest with any financial organization regarding the material discussed in the manuscript.

Authors' contributions.-OJK conceived and designed research; all authors conducted experiments; OJK, EF, KM, DP, RS, and JW analysed data; OJK wrote the manuscript; all authors read and approved the manuscript.

Acknowledgements.-The authors thank the subjects for participating and Weize Sun for expert help with experiments.

\section{TITLES OF FIGURES}

Figure 1.-A: Overview of high-intensity interval training (HIIT) protocols, with $4 \mathrm{x} 4$-minute high-intensity intervals (ON1-4, $\mathrm{n}=7$ ) set to correspond to $80 \%, 85 \%, 90 \%, 95 \%$, or $100 \%$ of maximal oxygen uptake $\left(\mathrm{VO}_{2 \max }\right)$ and $4 \mathrm{x} 4$-minute active recovery bouts $(\mathrm{OFF} 1-4, \mathrm{n}=7)$ set to correspond to $60 \%, 70 \%, 80 \%, 90 \%$, or $100 \%$ of lactate (La') threshold (LT), with the last OFF4-transient prolonged to allow full recovery. Oxygen uptake $\left(\mathrm{VO}_{2}\right)$ and heart rate $(\mathrm{HR})$ measurements in the last minute of each ON-transient interval confirmed intensity, whereas blood $\mathrm{La}^{-}$was sampled at the end of each ON-transient and every minute of OFF-transients. B: Sustainable duration during HIIT protocols performed at exercise intensities $80-100 \%$ of $\mathrm{VO}_{2 \max }$ C: Blood $\mathrm{La}^{-}$concentration ([La-]) during HIIT protocols performed at exercise intensities of $80-100 \%$ of $\mathrm{VO}_{2 \max }$. Statistically significantly different from other exercise 
intensities: ${ }^{* *} p<0.01, * p<0.05$. Statistically significantly different from baseline (BL): \#\# $p<0.01$, $\# p<0.05$.

Figure 2.-Rate of blood lactate $\left(\mathrm{La}^{-}\right)$concentration ([La $]$) rise during high-intensity intervals (ON-transients; A) and decrease (OFF-transients; B) of high-intensity interval training (HIIT) protocols, with ON-transients set to correspond to $80-100 \%$ of maximal oxygen uptake $\left(\mathrm{VO}_{2 \max }\right)$ of OFF-transients set to correspond to $60 \%$ of $\mathrm{La}^{-}$threshold (LT). Statistically significantly different from other intensities: ${ }^{* *} p<0.01$.

Figure 3.-Blood lactate $\left(\mathrm{La}^{-}\right)$concentration ([La-]) during high-intensity interval training (HIIT) protocols, with 4x4-minute high-intensity intervals (ON1-4) set to correspond to 95\% of maximal oxygen uptake $\left(\mathrm{VO}_{2 \max }\right)$, interspersed by $4 \mathrm{x} 4$-minute active recovery bouts set to correspond to $60 \%, 70 \%, 80 \%, 90 \%$, or $100 \%$ of $\mathrm{La}^{-}$threshold (LT; OFF1-4), with the last OFF4-transient prolonged to allow full recovery. BL: baseline. Statistically significantly different from OFF1 for active recovery intensities at $80-100 \%$ of LT, but not at $60-70 \%$ of LT: ${ }^{*} p<0.05$; statistically significantly different from other active recovery intensities: \# $p<0.05$.

Figure 4.-Lactate $\left(\mathrm{La}^{-}\right)$clearance of each active recovery intensity during the prolonged last post-interval active recovery bout (OFF4), with active recovery set to $60-100 \% \mathrm{of} \mathrm{La}^{-}$ threshold (LT); A: Time constants for 2/3 clearance, B: Maximal rate of $\mathrm{La}^{-}$clearance. Statistically significantly different from other active recovery intensities: ${ }^{*} p<0.05$. 